\title{
Effect of Topical Antibiotic Prophylaxis on Conjunctival Flora and Antibiotic Resistance Following Intravitreal Injections in Patients with Type 2 Diabetes
}

\author{
Havva Kaldirim¹, Serpil Yazgan², Ahmet Kirgiz ${ }^{3}$, Bilge Ozdemir ${ }^{4}$, Ahu Yilmaz ${ }^{1}$ \\ ${ }^{1}$ Department of Ophthalmology, Bagcilar Training and Research Hospital, Health Sciences University, Istanbul, Turkey \\ ${ }^{2}$ Department of Ophthalmology, Medical Faculty, Inonu University, Malatya, Turkey \\ ${ }^{3}$ Department of Ophthalmology, Beyoglu Eye Training and Research Hospital, Health Sciences University, Istanbul, Turkey \\ ${ }^{4}$ Department of Microbiology, Bagcilar Training and Research Hospital, Health Sciences University, Istanbul, Turkey
}

\begin{abstract}
Purpose: We sought to determine changes in the conjunctival bacterial flora and antibiotic resistance after topical antibiotic drops for infection prophylaxis were administered following intravitreal injections in patients with type 2 diabetes.

Methods: This prospective and nonrandomized cohort study included 116 eyes of 116 treatment-naive patients with type 2 diabetes who received six serial intravitreal anti-vascular endothelial growth factor injections for macular edema. Three conjunctival cultures were obtained from each eye over the course of the study (Culture 1, baseline; Culture 2, 1 month after the third injection; and Culture 3, 1 month after the sixth injection). The study subjects were given topical moxifloxacin hydrochloride for 4 days after each monthly intravitreal injection. The growth patterns of conjunctival bacterial flora and the antibiotic resistance to several commonly used antibiotics were examined.

Results: The rate of culture positivity increased significantly during the observation period (Culture 1, $n=47,40.5 \%$; Culture $2, n=58,50 \%$; Culture 3, $n=76,65.5 \%, p<0.001$ ). The bacterium with the highest baseline culture positivity was Staphylococcus epidermidis ( $n=45,38.8 \%)$, which increased significantly during the observation period $(p<0.001)$. No significant increase was noted in the culture positivity of the other bacteria with baseline culture positivity $(p>0.05)$. Regarding antibiotic susceptibility, significant increases in resistance to the fluoroquinolone group of drugs were noted $(p<0.001)$. No significant changes in sensitivity were detected in the other 11 investigated antibiotics that are commonly used in clinical practice $(p>$ 0.05).
\end{abstract}

Conclusions: The use of topical moxifloxacin after each intravitreal injection significantly increases the fluoroquinolone resistance of the ocular surface flora and the culture-positivity rate of S. epidermidis in patients with type 2 diabetes.

Key Words: Antibacterial drug resistance, Antibiotic prophylaxis, Diabetes mellitus, Intravitreal injections, Macular edema

Corresponding Author: Havva Kaldirim, MD. Department of Ophthalmology, Bagcilar Training and Research Hospital, Health Sciences University, Bagcilar Training and Research Hospital, Central Quarter, Dr. Sadik Ahmet Street, Bagcilar, Istanbul 34100, Turkey. Tel: 90-212-440-4000, Fax: 90-212440-4242, E-mail: : istanbuleah30@saglik.gov.tr

This study has been presented as a free paper at the EURETINA Congress, Vienna, 2018. 
The use of intravitreal injections (IVIs) has spread in recent years as a result of the increased effectiveness of anti-vascular endothelial growth factor (anti-VEGF) agents, which have proven to have played an important role in ophthalmologic diseases such as exudative age-related macular degeneration, retinal vein occlusion, and diabetic macular edema (DME) [1-3]. While IVI is considered to be an effective and safe method, endophthalmitis is the most feared complication, although its incidence after IVI is as low as $0.019 \%$ [4-6]. Even though there is a paucity of definitive evidence that demonstrates the correlation between the presence of ocular surface bacteria and the risk of developing endophthalmitis, it is believed that most bacteria isolated from patients with endophthalmitis after IVI are similar to the surface bacterial flora [7]. Several studies to date have identified an increased frequency of positive conjunctival cultures, differences in the identified microorganisms, and more pathogenic bacteria in diabetics relative to nondiabetics $[8,9]$.

Guidelines for minimizing the risk of endophthalmitis in the setting of IVI were established in 2004, although a consensus has yet to be reached regarding all methods of infection prophylaxis [10]. The use of topical povidone-iodine (PVI) on the ocular surface, eyelids, and eyelashes; the use of an eyelid speculum; and the avoidance of needle contact with surfaces other than the injection site have all been supported by a high level of agreement among expert reviewers. In contrast, the application of topical antibiotics, either before or after the injection procedure, has remained a topic of debate $[11,12]$. Although topical antibiotics have been proven to be effective in eliminating ocular surface bacteria in cataract surgical patients, even with the concomitant application of PVI, conclusions as to the nature of their role in IVI remain unclear and, to our knowledge, there no prior studies have systematically evaluated their efficacy in this clinical setting [13]. More than $80 \%$ of retinal specialists who responded to the 2009 American Society of Retinal Specialists Preferences and Trends survey reported using prophylactic topical antibiotics after IVI, although it remains unclear whether post-injection antibiotics offer any benefit, while recent evidence suggests that their use does not reduce the risk of endophthalmitis [14]. The indiscriminate use of topical antibiotics after such procedures may have the detrimental effect of provoking antibiotic resistance among the conjunctival bacterial flora [15].
This study aimed to evaluate changes in conjunctival bacterial flora and antibiotic resistance patterns in patients with type 2 diabetes who have received six serial IVIs following the post-injection use of topical antibiotics in the study eye.

\section{Materials and Methods}

\section{Overview}

This prospective, nonrandomized-cohort study was conducted at the University of Health Sciences Bagcilar Education and Research Hospital, Department of the Retina, between January 2016 and July 2017. Ethical approval was obtained from the Local Clinical Research Ethics Committee T. C. Public Hospitals Institution, Bagcilar Training and Research Hospital Non-Interventional Clinical Research Ethics Committee (project no. 2016-511) and this study was carried out according to the tenets of the Declaration of Helsinki. All subjects provided written informed consent at the time of enrollment.

The eyes of patients with newly diagnosed macular edema (ME) secondary type 2 diabetes mellitus who were undergoing treatment with intravitreal ranibizumab (Genentech, South San Francisco, CA) or aflibercept (Eylea; Regeneron Pharmaceuticals, Tarrytown, NY, USA) were deemed eligible for inclusion in this study if they had received at least six IVIs of their medication for DME and had used topical moxifloxacin hydrochloride $0.5 \%$ (Vigamox; Alcon Laboratories, Fort Worth, TX, USA) after each injection. The diagnosis of DME was determined according to the Early Treatment Diabetic Retinopathy Study criteria [16]. Patients who had received previous treatment with IVI, who had undergone previous ocular surgery, who had engaged in the chronic use of any topical ophthalmic medication, or who had used topical or systemic antibiotics within the past 6 months were excluded from this study. Patients who were unable to attend the scheduled follow-up appointments and complete the treatment were also excluded. After informed consent was obtained, the patients were enrolled in the study by a single retina specialist (HK).

A thorough ocular examination was completed at baseline and cultures were taken at baseline (Culture 1), 1 month after the third (Culture 2), and 1 month after the 
sixth (Culture 3) injections, respectively. All cultures were collected before the application of any topical drops or PVI. The IVI was administered in a standard fashion after obtaining informed consent and a culture. An injection kit from Blue Vision Company (Istanbul, Turkey) was used in all injections. The disposable materials included in each kit are one eye speculum, one $40-\times 40-\mathrm{cm}$ drape with a pouch, one $50 \times 50-\mathrm{cm}$ table drape, five cotton swabs, four pieces of gauze bandage, one 30-gauge cannula, one scleral marker, one 20-gauge cannula, one pair of gloves, and a $1-\mathrm{mL}$ injector. Anesthesia was achieved using topical proparacaine hydrochloride, $0.5 \%$ (Bausch and Lomb, Rochester, NY, USA). PVI (Betadine; Alcon Labs, Geneva, Switzerland) as $5 \%$ drops was applied, then a $10 \%$ PVI swab stick was painted over the injection site and applied to the ocular surface, making use of a sterile eye speculum and drape. Ranibizumab $0.5 \mathrm{mg}$ in $0.05 \mathrm{~mL}$ or aflibercept $2 \mathrm{mg}$ in $0.05 \mathrm{~mL}$ was injected 3.5 to $4 \mathrm{~mm}$ posterior to the limbus in the inferotemporal quadrant using a sterile 30 -gauge hypodermic needle in the operating room. Patients received topical moxifloxacin $0.5 \%$ four times per day for 4 days after each IVI, and this step was not altered even if antibiotic resistance was detected in the culture. As per the study protocol, patients were seen every month for 6 months, with ocular history-taking and examinations completed at each follow-up visit. Findings of endophthalmitis or serious adverse events were recorded and reviewed on a quarterly basis.

\section{Culture collection technique}

Before any eye drop or PVI application, conjunctival cultures were taken in a standardized fashion by swabbing the inferior conjunctival fornix using a BBL CultureSwab (Becton Dickinson, Franklin Lakes, NJ, USA). Care was taken to avoid contacting the eyelids or eyelashes with the swab. The swab samples were cultured on blood, chocolate, EMB agar, and MacConkey agar plates. Gram staining and testing for the presence or absence of catalase and coagulase/agglutination were performed to identify specific bacterial strains. The mean inhibitory concentration (MIC, $\mu \mathrm{g} / \mathrm{mL}$ ) for each fluoroquinolone was calculated using the Epsilometer test (bioMerieux, Marcy-l'Étoile, France), while the MICs for oxacillin, tetracycline, erythromycin, vancomycin, tobramycin, and other antibiotics were calculated using the double-disc diffusion test on the
Phoenix system (Becton Dickinson). Using the categories "susceptible" and "resistant," antibiotic sensitivity was determined from the MIC and organism data based on the guidelines of the National Committee for Clinical Laboratory Standards.

\section{Statistical analysis}

Statistical analyses were performed using the IBM SPSS Statistics ver. 19.0 (IBM Corp., Armonk, NY, USA). The distribution of data was determined with the Shapiro-Wilk test. Continuous variables were expressed as means \pm standard deviations, while categorical variables were expressed as frequencies and percentages. Continuous variables were compared using the Mann-Whitney $U$-test for two groups, while repeated measures were evaluated with a Friedman test for continuous variables. To compare for the frequency of related samples, a Cochran Q test was used, while Dunn test was adopted as a post-hoc test after the Cochrane Q test. A $p$-value of less than 0.05 was considered to be statistically significant in all tests.

\section{Results}

\section{Demographic characteristics}

A total of 116 phakic eyes belonging to 116 patients were enrolled in this study. The average age among the study subjects was $64.97 \pm 5.62$ years and $62(53.5 \%)$ were female and $54(46.5 \%)$ were male. The mean diabetic duration was $23.42 \pm 4.47$ years. All patients were given a total of six serial IVIs for DME in the study period, where 50 eyes (43.1\%) received ranibizumab and 66 eyes $(56.9 \%)$ received aflibercept. Table 1 summarizes the baseline demographics of the study population.

\section{Conjunctival culture results}

Culture 1 had the lowest positivity, while Culture 3 had the highest positivity (Culture 1, $\mathrm{n}=47,40.5 \%$; Culture 2, $\mathrm{n}=58,50 \%$; Culture 3, $\mathrm{n}=76,65.5 \%, p<0.001$ ) (Table 2). In the binary group comparison, no significant difference was identified between the Culture 1 and Culture 2 positivity rates ( $p=0.093$ ), while the positivity rate of Culture 3 was significantly higher than those of Cultures 1 and 2 
Table 1. Demographic features of the study group

\begin{tabular}{lc}
\hline Parameter & Value \\
\hline Female : male & $62(53.5): 54(46.5)$ \\
Age (yr) & $64.97 \pm 5.62$ \\
Diabetes mellitus (yr) & $23.42 \pm 4.47$ \\
Anti-VEGF type (R : A) & $50(43.1): 66(56.9)$ \\
\hline
\end{tabular}

Values are presented as number $(\%)$ or mean \pm standard deviation.

$\mathrm{VEGF}=$ vascular endothelial growth factor; $\mathrm{R}$ = ranibizumab; $\mathrm{A}=$ aflibercept. $\left(p_{1-3}<0.001, p_{2-3}=0.001\right)$ (Table 3$)$. The bacterium with the highest baseline culture positivity was coagulase-negative staphylococcus (Staphylococcus epidermidis, Culture 1, n $=45,38.8 \%$ ), and the culture positivity rate of this bacterium increased significantly during observation (Culture 2 , $\mathrm{n}=53,45.7 \%$; Culture $3, \mathrm{n}=74,63.8 \%, p<0.001)$. In the other bacteria with baseline culture positivity (S. aureus, Corynebacterium spp., Propionibacterium, Streptococcus spp., Klebsiella spp., Escherichia coli, Enterococcus faecalis, Stenotrophomonas maltophilia), no significant increase in culture positivity was observed during the follow-up period $(p>0.05)$ (Table 2).

Table 2. Changes in conjunctival bacterial flora and antibiotic susceptibility during the observation period

\begin{tabular}{|c|c|c|c|c|}
\hline & Culture $1(n=47)$ & Culture $2(\mathrm{n}=58)$ & Culture $3(n=76)$ & $p$-value ${ }^{*}$ \\
\hline Culture positivity & $47(40.5)$ & $58(50)$ & $76(65.5)$ & $<0.001$ \\
\hline Staphylococcus epidermidis & $45(38.8)$ & $53(45.7)$ & $74(63.8)$ & $<0.001$ \\
\hline Staphylococcus aureus & $9(7.8)$ & $13(11.2)$ & $13(11.2)$ & 0.264 \\
\hline Corynebacterium spp. & $16(13.8)$ & $17(14.7)$ & $9(7.8)$ & 0.051 \\
\hline Propionibacterium & $3(2.6)$ & $4(3.4)$ & $1(0.9)$ & 0.247 \\
\hline Streptococcus spp. & $11(9.5)$ & $13(11.2)$ & $18(15.5)$ & 0.307 \\
\hline Other bacteria ${ }^{\dagger}$ & $9(7.8)$ & $9(7.8)$ & $10(8.6)$ & 0.946 \\
\hline \multicolumn{5}{|c|}{ Antibiotic sensitivity (sensitive : resistant) } \\
\hline Ciprofloxacin & $40: 7(85: 15)$ & $29: 29(50: 50)$ & $21: 55(28: 72)$ & $<0.001$ \\
\hline Levofloxacin & $43: 4(92: 8)$ & $29: 29(50: 50)$ & $21: 55(28: 72)$ & $<0.001$ \\
\hline Moxifloxacin & $43: 4(92: 8)$ & $29: 29(50: 50)$ & $21: 55(28: 72)$ & $<0.001$ \\
\hline Amikacin & $45: 2(96: 4)$ & $56: 2(97: 3)$ & $74: 2(97: 3)$ & 0.368 \\
\hline Tobramycin & $45: 2(96: 4)$ & $56: 2(97: 3)$ & $72: 4(95: 5)$ & 0.058 \\
\hline Erythromycin & $33: 14(70: 30)$ & $43: 15(74: 26)$ & $57: 19(75: 25)$ & 0.472 \\
\hline Tetracycline & $31: 16(66: 34)$ & $40: 18(69: 31)$ & $50: 26(66: 34)$ & 0.368 \\
\hline Fusidic acid & $5: 42(11: 89)$ & $3: 55(5: 95)$ & $5: 71(7: 93)$ & 0.607 \\
\hline Penicillin G & $7: 40(18: 82)$ & $6: 52(10: 90)$ & $9: 67(12: 88)$ & 0.368 \\
\hline Oxacillin & $28: 19(60: 40)$ & $28: 30(48: 52)$ & $35: 41(46: 54)$ & 0.368 \\
\hline Ampicillin & $25: 22(53: 47)$ & $22: 36(38: 62)$ & $32: 44(42: 58)$ & 0.125 \\
\hline Linezolid & $42: 5(89: 11)$ & $53: 5(91: 9)$ & $70: 6(92: 8)$ & 0.368 \\
\hline Vancomycin & $47: 0(100: 0)$ & $58: 0(100: 0)$ & $76: 0(100: 0)$ & NA \\
\hline Clindamycin & $41: 6(87: 13)$ & $52: 6(90: 10)$ & $69: 7(91: 9)$ & 0.717 \\
\hline HbAlc $(\%)$ & $8.05 \pm 1.51$ & $8.14 \pm 1.64$ & $8.11 \pm 1.60$ & 0.213 \\
\hline
\end{tabular}

Values are presented as number (\%) or mean \pm standard deviation. Culture 1, baseline; Culture 2, 1 month after the third injection; Culture 3,1 month after the sixth injection.

$\mathrm{NA}=$ not available.

${ }^{*}$ Cochran Q test results. ${ }^{\dagger}$ Klebsiella spp., Escherichia coli, Enterococcus faecalis, and Stenotrophomonas maltophilia. 


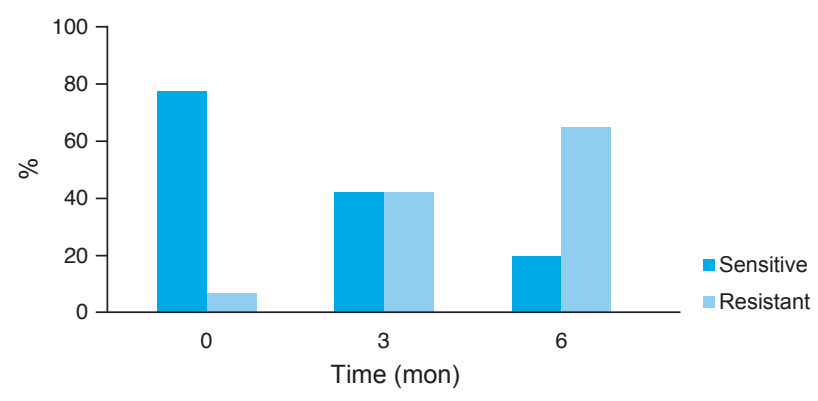

Fig. 1. Alteration of ciprofloxacin resistance during the observation period. It was observed that the sensitivity to ciprofloxacin decreased and the resistance increased over time.

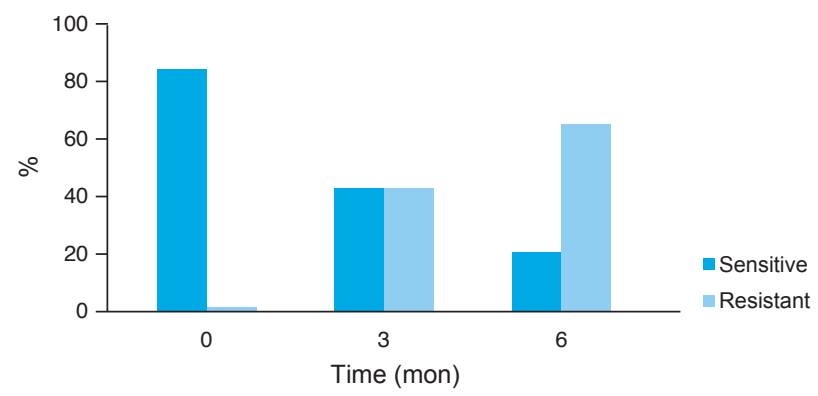

Fig. 2. Alteration of levofloxacin resistance during the observation period. It was observed that the sensitivity to levofloxacin decreased and the resistance increased over time.

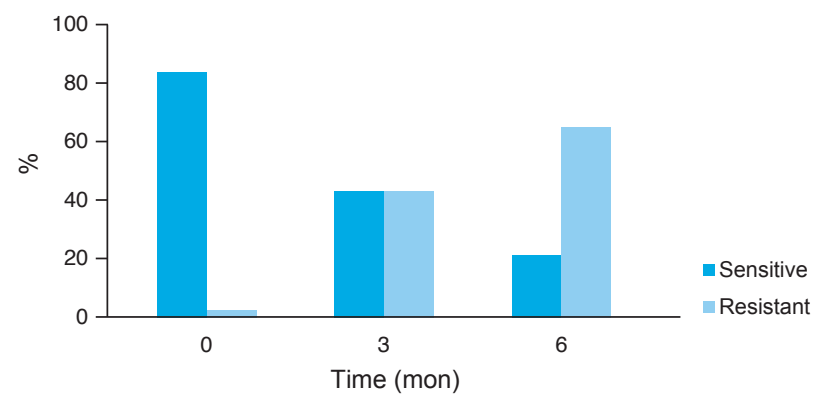

Fig. 3. Alteration of moxifloxacin resistance during the observation period. It was observed that the sensitivity to moxifloxacin decreased and the resistance increased over time.

\section{Results of culture antibiotic sensitivity}

Antibiotic susceptibility tests were performed separately for Cultures 1, 2, and 3 during this study. The rate of ciprofloxacin resistance was 15\% (7 / 47 cases) before IVI but increased significantly to $50 \%$ in Culture 2 (29/58 cases)
Table 3. The results of binary group comparison

\begin{tabular}{lccc}
\hline Parameter & $\begin{array}{c}p \text {-value } \\
(\text { Culture 1-2) }\end{array}$ & $\begin{array}{c}p \text {-value } \\
(\text { Culture 2-3) }\end{array}$ & $\begin{array}{c}p \text {-value } \\
\text { (Culture 1-3) }\end{array}$ \\
\hline Ciprofloxacin & 0.002 & 0.001 & $<0.001$ \\
Levofloxacin & $<0.001$ & 0.002 & $<0.001$ \\
Moxifloxacin & $<0.001$ & 0.002 & $<0.001$ \\
$\begin{array}{l}\text { Culture positivity } \\
\begin{array}{l}\text { Staphylococcus } \\
\text { epidermidis }\end{array}\end{array}$ & 0.093 & 0.001 & $<0.001$ \\
& 0.419 & $<0.001$ & $<0.001$
\end{tabular}

"Dunn test results (Cochran Q test with Dunn test as a post-hoc test).

and $72 \%$ in Culture 3 (55 / 76 cases) $(p<0.001)$ (Fig. 1). Separately, the rates of resistance to levofloxacin and moxifloxacin were similar before IVI ( $9 \%, 4$ / 47 cases) but also increased significantly and to a similar degree in the antibiogram of the second and third cultures (Culture 2, $50 \%$; Culture 3, 72\%; respectively; $p<0.001$ ) (Table 2, 3 and Fig. 2, 3). Meanwhile, there was no significant change in the rate of susceptibility to other investigated antibiotics (amikacin, tobramycin, erythromycin, tetracycline, fusidic acid, penicillin $\mathrm{G}$, oxacillin, ampicillin, linezolid, vancomycin, and clindamycin) $(p>0.05)$ (Table 2).

A significant correlation was found between the duration of diabetes mellitus and the positivity rate of Culture $3(p$ $=0.041)$ but not so with those of Cultures 1 or $2(p=0.814$ and $p=0.998$, respectively), while no significant change was witnessed in glycated hemoglobin (HbAlc) level during the follow-up period ( $p=0.213$ ) (Table 1). Thus, no correlation between culture positivity and $\mathrm{HbAlc}$ values was indicated. No adverse events were encountered in this study and no cases of endophthalmitis were identified.

\section{Discussion}

Intravitreal anti-VEGF injections have become a frequently adopted surgical procedure in ophthalmology clinics due to their efficacy in managing exudative age-related macular degeneration patients, ME secondary to diabetes mellitus, and retinal vascular occlusion. As with any intraocular surgery, the most daunting complication related to IVI is endophthalmitis. As endophthalmitis is hypothesized to occur through either the inoculation of bacteria into the eye at the time of injection or the passage of bacte- 
ria into the eye through a wound tract, sterilization of the ocular surface is paramount [17]. Several studies have demonstrated that the conjunctival flora in diabetics differs to a certain extent from that of nondiabetics $[8,9,18]$. For example, the frequency of positive conjunctival cultures has been found to be significantly higher in diabetics [18-20]. Thus, diabetic patients may be more predisposed to postoperative endophthalmitis [21]. Since the IVI of anti-VEGFs has become the treatment of choice for DME and this procedure is performed on a monthly basis in each patient, the number of diabetic individuals who develop post-injection infectious endophthalmitis is expected to increase. In our study, the positive conjunctival floral culture rates were $40.5 \%$ at baseline, $50 \%$ in the third month, and $65.5 \%$ in the 6th month. Martins et al. [8] reported culture positivity rates of $71.9 \%$ in eyes with proliferative diabetic retinopathy and $51.8 \%$ in eyes with non-proliferative diabetic retinopathy, which are higher values than our results. However, in their study, it is unclear whether the diabetic patients underwent intraocular surgery and IVI therapy or not prior to the study procedure. Thus, the high culture positivity rates of diabetic patients may be the result of conducting prophylactic topical antibiotic treatment previously. In our study, diabetic patients had never undergone any intraocular surgery procedure and had not received prophylactic antibiotic treatment before the study procedure. This may be the reason for the lower baseline culture positivity rate of our patients.

In a study conducted by Bilen et al. [9], the culture positivity rates were $65 \%$ in type 1 diabetic eyes, $78.8 \%$ in type 2 diabetic eyes, and $50 \%$ in control eyes, respectively. In this study, it is also uncertain whether the participants underwent intraocular surgery and used prophylactic antibiotics or not before entering the study. In summary, the baseline culture positivity rate (Culture 1) was lower in our study relative to in other studies $[8,9]$. The most likely causes of this outcome are that the diabetic patients who were included in our study never had any ocular surgery and never used prophylactic topical antibiotic therapy before the study protocol in contrast with in the abovementioned studies. In addition, differences in blood glucose regulation between patients (e.g., while the average Hbalc level of our cases was $8.1 \%$, the same was $9.2 \%$ in the study of Bilen et al. [9]) and environment and climate variations may affect the conjunctival floral positivity rate.

Consistent with the findings of Martins et al. [8], the present study also showed that the most prevalent microorganism in the conjunctival culture was coagulase-negative S. epidermidis. In the study conducted by Bilen et al. [9], the findings showed that $S$. epidermidis and $S$. aureus were the two bacteria most commonly isolated from the conjunctiva of patients with diabetes. In further retrospective studies involving patients who underwent cataract surgery, the findings reported greater prevalence rates of $S$. aureus, enterococci, certain streptococci (except Streptococcus pneumoniae) and Klebsiella spp. in the preoperative conjunctival bacterial cultures of diabetic patients [1820]. In the present study, however, the most frequently identified bacteria in the baseline culture was $S$. epidermidis (38.8\%), followed by Corynebacterium (13.8\%). Other breeding bacteria found in the baseline culture in the present study included Streptococcus spp., S. aureus, Klebsiella spp., enterococci, and Propionibacterium spp., in descending order of frequency. Coagulase-negative staphylococci and gram-negative bacteria have been described as the most common pathogens causing endophthalmitis in diabetic patients, while, following post-injection endophthalmitis, coagulase-negative staphylococci and streptococci are the most common causes of endophthalmitis after the IVI of anti-VEGF $[8,22,23]$.

There is currently a lack of consensus regarding the ideal IVI technique, pre-injection or post-injection care, and best-practice patterns. The most accepted prophylactic measure taken before IVI is the preparation of the injection site with topical PVI [6], although concerns have been raised related to the possibility that repeated exposure to PVI, as occurs with serial IVI, may lead to alterations in the conjunctival flora and perhaps even antibiotic resistance [24]. Supporting this theory are the results of some studies suggesting that other biocidal agents such as triclosan, chlorhexidine, and quaternary ammonium compounds may contribute to antibiotic cross-resistance [2527]. Although there is no evidence of bacterial resistance to PVI based on genomic mutation, the total effectiveness may be hampered by the fact that coagulase-negative staphylococci are frequently present in the conjunctiva in a biofilm, which may render them less susceptible to biocides and antibiotics [28]. While there is widespread belief that PVI does not lead to antibiotic resistance, the use of only PVI $5 \%$ at the time of the IVI, with no topical antibiotics, appears to have the lowest risk of contributing to the widespread problem of increasing antibiotic resistance 
$[14,24,29,30]$. Notably, the present study included no control group that used only PVI for prophylaxis; thus, resistance to direct PVI could not be evaluated. However, the culture antibiogram investigated 14 different antibiotics for resistance, observing significant resistance only in response to the fluoroquinolone group, while the prevalence rates of resistance to the other antibiotics investigated were similar to pre-injection rates, as shown in Table 2. Topical moxifloxacin prophylaxis was continued after IVI in 29 cases $(50 \%)$ who developed moxifloxacin resistance at the end of the second culture antibiogram in our study. However, no case of endophthalmitis had developed at the end of 6 months in any of the cases showing resistance. From this, we can conclude that antibiotic prophylaxis offers no additional advantage after anti-VEGF injections in the prevention of endophthalmitis in patients with DME. On the other hand, there is conflicting evidence related to the effects and the need for antibiotic prophylaxis in preventing post-injection endophthalmitis [6]. In a survey of IVI technique practice patterns among retinal specialists in the United States, findings showed that nearly one-third of participants administrate prophylactic topical antibiotics pre-injection - either for several days or immediately prior to IVI - while around $81 \%$ of doctors in the United States and $74 \%$ in the United Kingdom use prophylactic topical antibiotics post-injection [31]. Fluoroquinolones are the most commonly used topical antibiotics in ophthalmology [32,33], and when the currently available newer-generation fluoroquinolones became commercially available for ocular use, there was hope that their use would also aid in the prevention of post-procedure endophthalmitis [31]. Fluoroquinolones offer broad-spectrum antimicrobial coverage and good ocular penetration when used topically [34], although some studies have cautioned that endophthalmitis may still occur, despite the use of such agents $[35,36]$. The evidence is mounting regarding the risk of promoting resistant organisms on the ocular surface with widespread use of prophylactic antibiotics after IVI. Research has suggested that repeated exposure to fluoroquinolones-even via just a single drop on the eye after each injection - may lead to increased rates of resistance [28,37-39].

More recently, evidence has been emerging that suggests IVI could be performed safely without the need for antibiotic prophylaxis. The Diabetic Retinopathy Clinical Research Network reported no cases of endophthalmitis among 1,276 injections following a standardized injection protocol involving topical PVI, a sterile eyelid speculum, and topical anesthesia without the use of topical antibiotics [14]. Similarly, a study by Bhatt et al. [29] reported no significant difference in the rates of endophthalmitis after injection with or without antibiotics. However, although a several-day course of perioperative topical antibiotics may seem to be beneficial in reducing the risk of infection after IVI, current evidence does not support their use. The Diabetic Retinopathy Clinical Research Network reported a single case of endophthalmitis out of 3,333 injections without the use of topical antibiotics in comparison with six cases of endophthalmitis among 4,694 injections that were followed by a several-day course of post-injection prophylactic antibiotics [40]. Another study experienced nearly identical rates of endophthalmitis with the use of post-injection antibiotics (five cases among 2,287 injections; $0.22 \%$ ) and without the use of post-injection antibiotics (five cases among 2,480 injections; 0.20\%) [29].

Repeated, short 4-day courses of antibiotics have the potential for the selection of resistant bacterial strains. A short course may be enough to create a selection bias for resistance, although the duration may not be long enough for eradication. Repeated antibiotic use every 4 to 6 weeks enhances this selection force further and this could explain in part why fluoroquinolone resistance is emerging as a problem in ocular microbiology $[37,41]$.

The main source of bacteria isolated in cases of post-injection endophthalmitis is the patient's own conjunctival bacterial flora. In our study, we sought to evaluate changes in conjunctival bacterial flora and antibiotic resistance patterns in patients with type 2 diabetes following the administration of antibiotics after IVI. We opted to use a fourth-generation fluoroquinolone, moxifloxacin, which is a broad-spectrum bactericidal antibiotic that is active against both gram-positive and gram-negative bacteria. At the end of this study, we confirmed a significant increase in culture positivity after the third and sixth injections when compared with the baseline value.

One of the most important findings of this study is that the initial reproductive rate $(38.8 \%)$ of $S$. epidermidis, which is the most common endophthalmic agent in diabetic patients, increased to $63.8 \%$ at the end of the 6th month, while no significant increases were observed in the reproductive rates of other bacteria.

This study also investigated the relationship between HbAlc values and culture breeding rates, although no sig- 
nificant correlation was found between increased culture positivity and a higher HbAlc level. The rate of culture positivity at 6 months was found to be significantly greater in cases with a longer duration of diabetes mellitus, which may be attributed to the deterioration of the vascular system and weakening of the immune system present in patients with this condition.

One of the limitations of our study is the absence of a case group subjected only to PVI, while another limitation is that this study was carried out at a single center and, thus, features a relatively low number of cases. In this respect, the results obtained in this study should be supported by multicenter, large case series.

In conclusion, the results of our study demonstrate that a significant proportion of patients who are exposed to repeated short courses of topical fluoroquinolones after IVIs for DME develop ocular surface bacteria that are resistant to fluoroquinolones. Additional studies are needed to provide more definitive conclusions. However, the results of this study raise concerns about the growing rates of antibiotic resistance related to the routine use of topical antibiotics for endophthalmitis prophylaxis after repeated IVIs. Such a strategy may not reduce the risk of infection and may bring about changes in the conjunctival flora that may be potentially harmful by selecting for more antibiotic-resistant organisms.

\section{Conflict of Interest}

No potential conflict of interest relevant to this article was reported.

\section{Acknowledgements}

We would like to thank Furuzan Kokturk for assistance with statistical analysis in this study.

\section{References}

1. Rosenfeld PJ, Brown DM, Heier JS, et al. Ranibizumab for neovascular age-related maculardegeneration. $N$ Engl $J$ Med 2006;355:1419-31.

2. Brown DM, Campochiaro PA, Singh RP, et al. Ranibizum- ab for macular edema following central retinal vein occlusion: six-month primary end point results of a phase III study. Ophthalmology 2010;117:1124-33.

3. Massin P, Bandello F, Garweg JG, et al. Safety and efficacy of ranibizumab in diabetic macular edema (RESOLVE Study): a 12-month, randomized, controlled, double-masked, multicenter phase II study. Diabetes Care 2010;33:2399-405.

4. Scott IU, Flynn HW Jr. Reducing the risk of endophthalmitis following intravitreal injections. Retina 2007;27:10-2.

5. Pilli S, Kotsolis A, Spaide RF, et al. Endophthalmitis associated with intravitreal anti-vascular endothelial growth factor therapy injections in an office setting. Am J Ophthalmol 2008;145:879-82.

6. Cheung CS, Wong AW, Lui A, et al. Incidence of endophthalmitis and use of antibiotic prophylaxis after intravitreal injections. Ophthalmology 2012;119:1609-14.

7. de Caro JJ, Ta CN, Ho HK, et al. Bacterial contamination of ocular surface and needles in patients undergoing intravitreal injections. Retina 2008;28:877-83.

8. Martins EN, Alvarenga LS, Hofling-Lima AL, et al. Aerobic bacterial conjunctival flora in diabetic patients. Cornea 2004;23:136-42.

9. Bilen $\mathrm{H}$, Ates $\mathrm{O}$, Astam $\mathrm{N}$, et al. Conjunctival flora in patients with type 1 or type 2 diabetes mellitus. Adv Ther 2007;24: 1028-35.

10. Jager RD, Aiello LP, Patel SC, Cunningham ET Jr. Risks of intravitreous injection: a comprehensive review. Retina 2004;24:676-98.

11. Scott IU, Flynn HW Jr. The role of topical antibiotic prophylaxis for intravitreal injections. Arch Ophthalmol 2007;125:974-6.

12. Ta CN. Topical antibiotic prophylaxis in intraocular injections. Arch Ophthalmol 2007;125:972-4.

13. Ta CN, Egbert PR, Singh K, et al. Prospective randomized comparison of 3-day versus 1-hour preoperative ofloxacin prophylaxis for cataract surgery. Ophthalmology 2002;109: 2036-40.

14. Bhavsar AR, Googe JM Jr, Stockdale CR, et al. Risk of endophthalmitis after intravitreal drug injection when topical antibiotics are not required: the diabetic retinopathy clinical research network laser-ranibizumab-triamcinolone clinical trials. Arch Ophthalmol 2009;127:1581-3.

15. Kim SJ, Toma HS. Ophthalmic antibiotics and antimicrobial resistance a randomized, controlled study of patients undergoing intravitreal injections. Ophthalmology 2011;118:1358-63. 
16. Photocoagulation for diabetic macular edema. Early Treatment Diabetic Retinopathy Study report number 1. Early Treatment Diabetic Retinopathy Study research group. Arch Ophthalmol 1985;103:1796-806.

17. Green-Simms AE, Ekdawi NS, Bakri SJ. Survey of intravitreal injection techniques among retinal specialists in the United States. Am J Ophthalmol 2011;151:329-32.

18. Fernandez-Rubio ME, Rebolledo-Lara L, Martinez-Garcia $\mathrm{M}$, et al. The conjunctival bacterial pattern of diabetics undergoing cataract surgery. Eye (Lond) 2010;24:825-34.

19. Suto C, Morinaga M, Yagi T, et al. Conjunctival sac bacterial flora isolated prior to cataract surgery. Infect Drug Resist 2012;5:37-41.

20. Fernandez-Rubio ME, Cuesta-Rodriguez T, Urcelay-Segura JL, Cortes-Valdes C. Pathogenic conjunctival bacteria associated with systemic co-morbidities of patients undergoing cataract surgery. Eye (Lond) 2013;27:915-23.

21. Doft BH, Wisniewski SR, Kelsey SF, et al. Diabetes and postoperative endophthalmitis in the endophthalmitis vitrectomy study. Arch Ophthalmol 2001;119:650-6.

22. Durand ML. Endophthalmitis. Clin Microbiol Infect 2013;19:227-34.

23. Moshfeghi AA, Rosenfeld PJ, Flynn HW Jr, et al. Endophthalmitis after intravitreal vascular [corrected] endothelial growth factor antagonists: a six-year experience at a university referral center. Retina 2011;31:662-8.

24. Milder E, Vander J, Shah C, Garg S. Changes in antibiotic resistance patterns of conjunctival flora due to repeated use of topical antibiotics after intravitreal injection. Ophthalmology 2012;119:1420-4.

25. Bloomfield SF. Significance of biocide usage and antimicrobial resistance in domiciliary environments. $J$ Appl Microbiol 2002;92 Suppl:144S-57S.

26. Chuanchuen R, Beinlich K, Hoang TT, et al. Cross-resistance between triclosan and antibiotics in Pseudomonas aeruginosa is mediated by multidrug efflux pumps: exposure of a susceptible mutant strain to triclosan selects $\mathrm{nfxB}$ mutants overexpressing MexCD-OprJ. Antimicrob Agents Chemother 2001;45:428-32.

27. Russell AD. Biocide use and antibiotic resistance: the relevance of laboratory findings to clinical and environmental situations. Lancet Infect Dis 2003;3:794-803.

28. Ta CN, Chang RT, Singh K, et al. Antibiotic resistance patterns of ocular bacterial flora: a prospective study of patients undergoing anterior segment surgery. Ophthalmology 2003;110:1946-51.
29. Bhatt SS, Stepien KE, Joshi K. Prophylactic antibiotic use after intravitreal injection: effect on endophthalmitis rate. Retina 2011;31:2032-6.

30. Lanker Klossner B, Widmer HR, Frey F. Nondevelopment of resistance by bacteria during hospital use of povidone-iodine. Dermatology 1997;195 Suppl 2:10-3.

31. Samia-Aly E, Cassels-Brown A, Morris DS, et al. A survey of UK practice patterns in the delivery of intravitreal injections. Ophthalmic Physiol Opt 2015;35:450-4.

32. Chang DF, Braga-Mele R, Mamalis N, et al. Prophylaxis of postoperative endophthalmitis after cataract surgery: results of the 2007 ASCRS member survey. J Cataract Refract Surg 2007;33:1801-5.

33. Leaming DV. Practice styles and preferences of ASCRS members: 2000 survey. American Society of Cataract and Refractive Surgery. J Cataract Refract Surg 2001;27:948-55.

34. Hariprasad SM, Blinder KJ, Shah GK, et al. Penetration pharmacokinetics of topically administered $0.5 \%$ moxifloxacin ophthalmic solution in human aqueous and vitreous. Arch Ophthalmol 2005;123:39-44.

35. Moshirfar M, Feiz V, Vitale AT, et al. Endophthalmitis after uncomplicated cataract surgery with the use of fourth-generation fluoroquinolones: a retrospective observational case series. Ophthalmology 2007;114:686-91.

36. Deramo VA, Lai JC, Fastenberg DM, Udell IJ. Acute endophthalmitis in eyes treated prophylactically with gatifloxacin and moxifloxacin. Am J Ophthalmol 2006;142:721-5.

37. Park SH, Lim JA, Choi JS, et al. The resistance patterns of normal ocular bacterial flora to 4 fluoroquinolone antibiotics. Cornea 2009;28:68-72.

38. Kim SJ, Toma HS, Midha NK, et al. Antibiotic resistance of conjunctiva and nasopharynx evaluation study: a prospective study of patients undergoing intravitreal injections. Ophthalmology 2010;117:2372-8.

39. Moss JM, Sanislo SR, Ta CN. Antibiotic susceptibility patterns of ocular bacterial flora in patients undergoing intravitreal injections. Ophthalmology 2010;117:2141-5.

40. Bhavsar AR, Stockdale CR, Ferris FL 3rd, et al. Update on risk of endophthalmitis after intravitreal drug injections and potential impact of elimination of topical antibiotics. Arch Ophthalmol 2012;130:809-10.

41. Fintelmann RE, Hoskins EN, Lietman TM, et al. Topical fluoroquinolone use as a risk factor for in vitro fluoroquinolone resistance in ocular cultures. Arch Ophthalmol 2011;129:399402. 